\title{
Stochastic Coagulation Equation and Validity of the Statistical Coagulation Equation
}

\author{
Hidekazu TANAKA and Kiyoshi NAKAzAWA \\ Department of Earth and Planetary Sciences, Faculty of Science, \\ Tokyo Institute of Technology, Tokyo 152, Japan
}

(Received September 18, 1992; Revised February 5, 1993)

In the study of the origin of the solar system, many works on the coagulation process (i.e., the growth of dust grains and of planetesimals) have been made by using the coagulation equation which governs the time variation of the size distribution of "particles" due to collisional coalescence. As is conjectured by many authors, however, the coagulation equation itself may have a serious defect in some cases and, hence, cannot describe precisely the coagulation process; the essence of the defect lies on a fact that the coagulation equation inevitably creates particles with infinite mass. The purpose of the present study is to examine the conditions under which the coagulation equation can be regarded to be valid.

First, on the basis of the stochastic viewpoint, we derived a fundamental equation (called 'the stochastic coagulation equation') which describes exactly the coagulation process in a finite particle system. Then, investigating the properties of the stochastic coagulation equation, we found that it reduces to the ordinary coagulation equation if the correlation between the numbers of particles with masses $i$ and $j$ (i.e., $\left\langle n_{i} n_{j}\right\rangle-\left\langle n_{i}\right\rangle\left\langle n_{j}\right\rangle$ ) is negligibly small compared with $\left\langle n_{i}\right\rangle\left\langle n_{j}\right\rangle\left(n_{i}\right.$ being the number of particles with mass $i$ and brackets mean the ensemble average) and if the total particle number is sufficiently large.

Furthermore, we solved the stochastic coagulation equation for the three special cases where the coalescence rate $A_{i j}$ is given by $1, i+j$, and $i \times j$ and obtained its analytic solutions. Using the solutions of the latter two cases, we found that the ordinary coagulation equation, at least, can approximately describe the number of particles of which mass is much smaller than the geometrical mean of the smallest mass and the total mass of the particle system.

\section{Introduction}

It is generally believed that the terrestrial planets and the cores of the Jovian-type planets were formed from a large number of planetesimals through their successive mutual collisions and coalescences (e.g., HAYASH et al., 1985; Wetherill, 1980). In the Earth's region of the solar nebula, for example, there were about $1 \times 10^{10}$ planetesimals with masses of the order of $1 \times 10^{18}$ $\mathrm{g}$ which mutually collided and finally accumulated to the Earth. To describe mathematically, the accumulation process of such numerous planetesimals, statistical treatment is indispensable.

In the statistical treatment, the common use has been made of the coagulation equation, which governs the time evolution of planetesimals' mass distribution function (e.g., GreENBERG et al., 1978; OHtsuki et al., 1988; Wetherill and Stewart, 1989). The coagulation equation is written as

$$
\frac{d N_{k}(t)}{d t}=\frac{1}{2} \sum_{\substack{i, j=1 \\ i+j=k}} A_{i j} N_{i}(t) N_{j}(t)-N_{k}(t) \sum_{i=1}^{\infty} A_{k i} N_{i}(t)
$$


where $N_{k}(t)$ is the number of particles with mass of $k m_{0}$ at time $t$ ( $m_{0}$ being a unit mass, or in other words, the minimum mass of a particle system considered) and $A_{i j}$ is the coalescence rate between two particles with masses $i m_{0}$ and $j m_{0}$. The coalescence rate $A_{i j}$ is given by

$$
A_{i j}=\frac{\overline{\sigma_{i j} v_{i j}}}{V}
$$

where $\sigma_{i j}, v_{i j}$, and $V$ are the coalescent cross section between particles with masses $i m_{0}$ and $j m_{0}$, the relative velocity between them, and the volume of the system, respectively. Furthermore, the bar " - " denotes an appropriate mean with respect to the velocity distribution function of the particles. In Eq. (1), the first term on the right-hand side describes the increment of the number of particles $N_{k}(t)$ due to the coalescence of smaller particles and the resultant formation of particle with mass $k m_{0}$ : it is obtained by multiplying the coalescence rate $A_{i j}$ by the number of the combinations $N_{i}(t) N_{j}(t)$ and taking a sum over all pairs of masses $i m_{0}$ and $j m_{0}$ which create particles with mass $k m_{0}$. In the same way, the second term describes the decrease of $N_{k}(t)$ due to the coalescence between particles with mass $\mathrm{km}_{0}$ and others.

Although the coagulation equation (1) obtained in such a way seems reasonable at first sight, it actually has some essential defects. To see it, we consider a particle system in which all particles have initially a unit mass $m_{0}$. According to the coagulation equation (1), particles with masses larger than $m_{0}$ are created through the mutual collisions. The appearance of such large particles enables the creation of still larger particles, and finally, for an arbitrary large mass $k$ (for simplicity, we put $m_{0}$ to be unity from now on), the numbers $N_{k}$ become non-zero: the coagulation equation artificially creates particles with infinite mass, which is, of course, larger than the total mass of the particle system. Such an unphysical situation is due to the intrinsic defects of the coagulation equation. The major defect is found in the way of counting the combinations of two colliding particles: collisions between a particle and itself are also included. In the coagulation equation such a way of counting cannot be improved. Since the number of particles $N_{k}$ have non-integral value, it is impossible to count the combination correctly.

We show another example of breakdown of the coagulation equation. Analytic solutions to the coagulation equation have been found for several special cases with a simple form of the coalescence rate, i.e., $A_{i j}=$ constant (Smoluchowski, 1916), $A_{i j} \propto i+j$ (Trubnikov, 1971), and $A_{i j} \propto i \times j$ (Trubnikov, 1971). Among these cases, the last one is an extreme example where the coagulation equation breaks down because of the defects mentioned above. Noting that the total mass of the particle system $M_{T}$ is represented by the sum $\sum k N_{k}$, for the case of $A_{i j}=i \times j$, we can rewrite the coagulation equation (1) as

$$
\frac{d N_{k}(t)}{d t}=\frac{1}{2} \sum_{i+j=k} i j N_{i}(t) N_{j}(t)-k N_{k}(t) M_{T} .
$$

Assuming that all particles have a unit mass initially (i.e., $N_{k}(0)=M_{T} \delta_{k 1}$ ), the solution to Eq. (3) can be obtained as (Trubnikov, 1971)

$$
N_{k}(\tau)=M_{T} \frac{k^{k-2}}{k !} \tau^{k-1} \mathrm{e}^{-k \tau}
$$

where

$$
\tau=M_{T} t
$$

In the derivation of this solution, it was postulated that the total mass $M_{T}$ is constant. On the other hand, the total mass can be readily calculated from the above solution. Figure 1 shows the time-variation of the total mass. The total mass is conserved till $\tau=1$, but it gradually decreases 


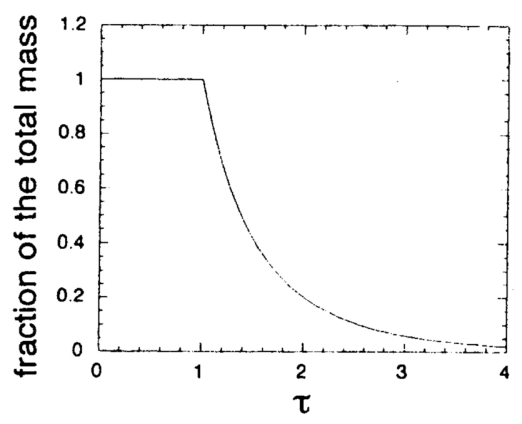

Fig. 1. The time-variation of the total mass calculated from the solution to the coagulation equation for the case of $A_{i j}=i \times j$. The total mass is conserved until $\tau=1$, but it decreases after $\tau=1$.

after $\tau=1$ : the mass conservation breaks down after $\tau=1$. WeTHERILL (1990) considered this contradiction as an appearance of a "runaway body", of which mass is determined so as to make the mass conservation hold. However, his prescription is not rigorous mathematically. The breakdown of the mass conservation is attributed to the defects of the coagulation equation itself, and this can quite easily be shown. Multiplying Eq. (3) by mass $k$ and taking a sum over mass $k$, we obtain the equation governing the total mass of the particle system:

$$
\frac{d M_{T}}{d t}=\sum_{k=1}^{\infty} \sum_{i+j=k} i^{2} j N_{i}(t) N_{j}(t)-M_{T} \sum_{k=1}^{\infty} k^{2} N_{k}(t) .
$$

If the two terms of the right-hand side are identical, the total mass is conserved. Assume that the sum

$$
\sum_{i=1}^{\infty} i^{2} N_{i}(t)
$$

is convergent. Then, the second term on the right-hand side can be rewritten as

$$
\begin{aligned}
M_{T} \sum_{i=1}^{\infty} i^{2} N_{i}(t) & =\sum_{i=1}^{\infty} \sum_{j=1}^{\infty} j N_{j}(t) i^{2} N_{i}(t) \\
& =\sum_{k=1}^{\infty} \sum_{i+j=k} i^{2} j N_{i}(t) N_{j}(t)
\end{aligned}
$$

and the right-hand side of Eq. (6) vanishes. Thus, as long as the sum $\sum k^{2} N_{k}(t)$ is convergent, the total mass is conserved. However, calculating the sum $\sum k^{2} N_{k}(t)$ directly from the solution (4), we can readily make sure of it's divergence at $\tau=1$. In the real finite system such a divergence cannot occur. The number of particles $N_{k}$ always vanishes for the mass $k$ greater than the total mass of the system, which makes the sum a finite series. Hence, it is concluded that the breakdown of the mass conservation is due to the artificial formation of infinite large particles.

Since the coagulation equation has, in some cases, obviously serious defects, it is important to find out under what condition the coagulation equation can correctly describe the accumulation process. In the early stage of accumulation of the planets where there were a large number of planetesimals with small masses, the statistical coagulation equation would be valid, whereas in the very late stage of the accumulation it would break down because of a small number of planetesimals. Unfortunately, we cannot tell when the coagulation equation becomes invalid in the course of the accumulation. 
In the present paper, at first, on the basis of the stochastic viewpoint, we will derive a fundamental equation which exactly describes the accumulation process (we will call the obtained equation the stochastic coagulation equation). Secondly, by solving the stochastic coagulation equation for typical cases, we clarify what conditions are needed for the stochastic coagulation equation to reduce to the ordinary, statistical coagulation equation (1). In the next section we will introduce the stochastic coagulation equation as a basic equation for the accumulation process, and in Section 3, analytic solutions to the stochastic coagulation equation will be presented for three kinds of coalescence rates (i.e., $A_{i j}=$ constant, $i+j$, and $i \times j$ ). In the final section, we will summarize our present study and discuss remaining problems to be solved.

\section{Stochastic Coagulation Equation}

\subsection{Derivation of the stochastic coagulation equation}

Generally, the accumulation process of planetesimals contains various kinds of mechanical and physical processes. For example, impact experiments in laboratory indicate that there are wide varieties in outcomes of collision (e.g., FuJIWARA and TAKAGI, 1988); when their colliding velocity is very large, two bodies collide to fragment catastrophically into a large number of small pieces. Frequently, collision leads to a coalescence with mass loss through impact jet. As is also known, the planetary accumulation (collision) process is inevitably accompanied by the migration and diffusion (toward and against the protosun) of planetesimals due to mutual gravitational scattering.

As mentioned in Section 1, however, the primary aim of the present work is to ascertain the validity of the ordinary statistical coagulation equation. In order to achieve this aim, apart from the complicated, realistic accumulation process, it is convenient to consider a simplified model where the following assumptions are justified:

1. Three- and many-particle collisions can be neglected.

2. Collision between two particles leads to their coalescence without mass loss.

3. The rate of coalescence $A_{i j}$ between $i$-th and $j$-th particles is completely determined only by physical variables of the colliding two particles (such as the mass, the radius, and the velocity) and free from those of the other particles.

4. The system consists of the finite number of particles (i.e., the total mass is finite).

Assumptions (1) to (3) are also made in deriving the ordinary coagulation equation, while the last one is peculiar to the present problem.

Now, we consider a system of particles whose total mass is $N$ (the unit mass of the particle is put to be unity and, then, the particle's mass is given by an integer from 1 to $N$ ). The mass distribution of the particles is therefore completely assigned by giving the number $n_{i}$ of particles with mass $i$, i.e., $n_{1}, n_{2}, \ldots, n_{N}$. In other words, the state of the mass distribution of the particle system is expressed by a $N$-dimensional vector $\mathbf{n}=\left(n_{1}, \ldots, n_{N}\right)$. We call it a state vector.

Let us introduce a function $f(\mathbf{n} ; t)$ giving a probability that the system is in a state $\mathbf{n}$ at time $t$. Then, from the conservation of the total probability, $f(\mathbf{n} ; t)$ must satisfy the relation

$$
\sum_{\mathbf{n}} f(\mathbf{n} ; t)=1
$$

at any time, where the sum is taken over all states. It is also noted that the total mass must be conserved and that the particle number $n_{i}$ is not negative for any mass $i$. Thus, we have

$$
\sum_{i=1}^{N} i n_{i}=N
$$


and

$$
n_{i} \geq 0, \quad \text { for all } i .
$$

For the unphysical state $\mathbf{n}^{\prime}$ violating equations (10) and/or (11), the probability function $f\left(\mathbf{n}^{\prime} ; t\right)$ must be zero:

$$
f\left(\mathbf{n}^{\prime} ; t\right)=0, \quad \text { for any unphysical state } \mathbf{n}^{\prime} .
$$

Now, we seek the equation which describes the time variation of the probability function $f(\mathbf{n} ; t)$. We suppose that two particles with masses $i$ and $j$ collide to form a single particle with mass $i+j$. Using the coalescence rate $A_{i j}$, the number of coalescence per unit time is given by

$$
\begin{cases}A_{i j} n_{i} n_{j}, & \text { for } i \neq j \\ A_{i i} \frac{n_{i}\left(n_{i}-1\right)}{2}, & \text { for } i=j\end{cases}
$$

Thus, the equation to be obtained is found as follows:

$$
\begin{aligned}
\frac{\partial}{\partial t} f(\mathbf{n} ; t)= & \sum_{\substack{i, j=1 \\
i \neq j}}^{N} \frac{1}{2} A_{i j}\left(n_{i}+1\right)\left(n_{j}+1\right) f\left(\ldots, n_{i}+1, \ldots, n_{j}+1, \ldots, n_{i+j}-1, \ldots ; t\right) \\
& +\sum_{i=1}^{N} \frac{1}{2} A_{i i}\left(n_{i}+2\right)\left(n_{i}+1\right) f\left(\ldots, n_{i}+2, \ldots, n_{2 i}-1, \ldots ; t\right) \\
& -\sum_{\substack{i, j=1 \\
i \neq j}}^{N} \frac{1}{2} A_{i j} n_{i} n_{j} f(\mathbf{n} ; t) \\
& -\sum_{i=1}^{N} \frac{1}{2} A_{i i} n_{i}\left(n_{i}-1\right) f(\mathbf{n} ; t) .
\end{aligned}
$$

The first two sums in the right-hand side represent the probabilities of transitions from other states into a state $\mathbf{n}$ by the coalescences between particles $i$ and $j$ and between particles of the same mass $i$. On the other hand, the remaining two sums represent the probabilities of transitions from the state $\mathbf{n}$ to other states. This is the basic stochastic equation describing the growth of particles exactly. Note that each sum in the right-hand side of this equation includes unnecessary terms where $i+j>N$ : these terms represent unphysical collisions which create particles with mass larger than the total mass. However, as readily seen, such terms always vanish owing to the condition (12). Accordingly, Eq. (14), in effect, forbids such unphysical collisions.

For the later convenience (the above expression is expensively complicated and inconvenient for further analysis), we will rewrite it in a simple form. Let $a_{i}^{\dagger}$ and $a_{i}$ be the generation operator and extinction operator, respectively, which act as

$$
\left\{\begin{array}{l}
a_{i}^{\dagger} Q\left(n_{i}\right)=Q\left(n_{i}+1\right) \\
a_{i} Q\left(n_{i}\right)=Q\left(n_{i}-1\right)
\end{array}\right.
$$

where $Q\left(n_{i}\right)$ is an arbitrary function of $n_{i}$. By the use of these operators, Eq. (14) can be rewritten in the following form:

$$
\frac{\partial}{\partial t} f(\mathbf{n} ; t)=\sum_{i, j=1}^{N} \frac{1}{2} A_{i j}\left[a_{i+j} a_{i}^{\dagger} n_{i} a_{j}^{\dagger} n_{j}-n_{i} a_{i} n_{j} a_{i}^{\dagger}\right] f(\mathbf{n} ; t) .
$$


Before we go on to the next step of our study, we will see that the stochastic coagulation equation obtained above ensures the conservation of the total probability. We first introduce some useful auxiliary equations. Let $Q$ be a function which is independent of $n_{i}, a_{i}$, and $a_{i}^{\dagger}$. Then, for an integer $p$ the following two equations can be readily obtained:

$$
\begin{cases}\sum_{\mathbf{n}} a_{i}^{\dagger} n_{i}^{p} Q f(\mathbf{n} ; t)=\sum_{\mathbf{n}} n_{i}^{p} Q f(\mathbf{n} ; t) & \text { for } p \geq 1, \\ \sum_{\mathbf{n}} a_{i} n_{i}^{p} Q f(\mathbf{n} ; t)=\sum_{\mathbf{n}} n_{i}^{p} Q f(\mathbf{n} ; t) & \text { for } p \geq 0 .\end{cases}
$$

Furthermore, from the definition of the operators (15), we can obtain the following commutation rules for the operators $a_{i}^{\dagger}, a_{i}$, and a function $Q\left(n_{j}\right)$ :

$$
\left\{\begin{array}{l}
a_{i}^{\dagger} Q\left(n_{j}\right)=Q\left(n_{j}+\delta_{i j}\right) a_{i}^{\dagger} \\
a_{i} Q\left(n_{j}\right)=Q\left(n_{j}-\delta_{i j}\right) a_{i}
\end{array}\right.
$$

where $\delta_{i j}$ is the Kronecker delta. These auxiliary equations will be frequently referred to later. To see the conservation of the total probability, we now take a sum of Eq. (16) over all possible states:

$$
\frac{\partial}{\partial t} \sum_{\mathbf{n}} f(\mathbf{n} ; t)=\sum_{i, j=1}^{N} \frac{1}{2} A_{i j} \sum_{\mathbf{n}}\left[a_{i+j} a_{i}^{\dagger} n_{i} a_{j}^{\dagger} n_{j}-n_{i} a_{i} n_{j} a_{i}^{\dagger}\right] f(\mathbf{n} ; t) .
$$

Using Eqs. (17) and (18), the first term in the parentheses of Eq. (19) is transformed as follows:

$$
\begin{aligned}
\sum_{\mathbf{n}} a_{i+j} a_{i}^{\dagger} n_{i} a_{j}^{\dagger} n_{j} f(\mathbf{n} ; t) & =\sum_{\mathbf{n}} a_{i+j} a_{i}^{\dagger} a_{j}^{\dagger}\left(n_{i}-\delta_{i j}\right) n_{j} f(\mathbf{n} ; t) \\
& =\sum_{\mathbf{n}}\left(n_{i}-\delta_{i j}\right) n_{j} f(\mathbf{n} ; t) .
\end{aligned}
$$

Moreover, because $a_{i} a_{i}^{\dagger}=1$, we can rewrite the second term in the parentheses as follows:

$$
\begin{aligned}
\sum_{\mathbf{n}} n_{i} a_{i} n_{j} a_{i}^{\dagger} f(\mathbf{n} ; t) & =\sum_{\mathbf{n}} n_{i}\left(n_{j}-\delta_{i j}\right) a_{i} a_{i}^{\dagger} f(\mathbf{n} ; t) \\
& =\sum_{\mathbf{n}} n_{i}\left(n_{j}-\delta_{i j}\right) f(\mathbf{n} ; t)
\end{aligned}
$$

Thus, the right-hand side of Eq. (19) vanishes identically and, hence, the total probability is conserved.

\subsection{Reduction to the ordinary coagulation equation}

Let us denote an expectation of a stochastic variable $n_{k}$ by $\left\langle n_{k}\right\rangle$ :

$$
\left\langle n_{k}\right\rangle \equiv \sum_{\mathbf{n}} n_{k} f(\mathbf{n} ; t)
$$

It should be noted that $\left\langle n_{k}\right\rangle$ corresponds to $N_{k}(t)$ which appears in the ordinary coagulation equation (1). Thus, in order to directly compare these two equations (i.e., the ordinary statistical and the stochastic coagulation equations), we will seek an alternative expression of the stochastic coagulation equation (16), which describes the time variation of $\left\langle n_{k}\right\rangle$. 
Multiplying Eq. (16) by $n_{k}$ and taking a sum over all states, we obtain

$$
\begin{aligned}
\frac{\partial}{\partial t}\left\langle n_{k}\right\rangle & =\sum_{\mathbf{n}} n_{k} \frac{\partial}{\partial t} f(\mathbf{n} ; t) \\
& =\sum_{i, j=1}^{N} \frac{1}{2} A_{i j} \sum_{\mathbf{n}}\left[\left(n_{k}+\delta_{k, i+j}-\delta_{k i}-\delta_{k j}\right)\left(n_{i}-\delta_{i j}\right) n_{j}-n_{k} n_{i}\left(n_{j}-\delta_{i j}\right)\right] f(\mathbf{n} ; t),
\end{aligned}
$$

where we used Eqs. (17) and (18). After a simple calculation, we finally obtain

$$
\frac{\partial}{\partial t}\left\langle n_{k}\right\rangle=\frac{1}{2} \sum_{i, j=1}^{N} A_{i j} \delta_{k, i+j}\left\langle n_{i}\left(n_{j}-\delta_{i j}\right)\right\rangle-\sum_{i=1}^{N} A_{i k}\left\langle n_{k}\left(n_{i}-\delta_{i k}\right)\right\rangle
$$

where the brackets \langle\rangle denote the same meaning as that of Eq. (22). This equation is very similar to but slightly different from the ordinary coagulation equation (1). These two equations coincide with each other only in the cases when $\left\langle n_{i}\left(n_{j}-\delta_{i j}\right)\right\rangle$ can be replaced by $\left\langle n_{i}\right\rangle\left\langle n_{j}\right\rangle$, i.e.,

$$
\left\langle n_{i}\left(n_{j}-\delta_{i j}\right)\right\rangle=\left\langle n_{i}\right\rangle\left\langle n_{j}\right\rangle, \quad \text { for all } i \text { and } j \text {. }
$$

From the viewpoint of practical use (i.e., for the numerical simulation of the accumulation process of planetesimals), it is very important to make clear under what conditions the above relation (25) can be satisfied within a good accuracy. To answer this question, we have to investigate extensively the properties of solutions to the stochastic coagulation equation.

\subsection{The factorial moment equation}

Although the stochastic coagulation equation (16) completely determines the probabilities of all states, the quantities we want to know are the averaged ones of stochastic variables such as $\left\langle n_{i}\right\rangle$ and $\left\langle n_{i} n_{j}\right\rangle$, rather than the state probabilities themselves. Thus, it is convenient to rewrite the stochastic coagulation equation into the time-development equation of such quantities, i.e., the moment equations. An example of such equations (i.e., the first moment equation) was derived in the preceding subsection (see Eq. (24)). The first moment equations, however, are not complete mathematically because they include the second moments $\left\langle n_{i}\left(n_{j}-\delta_{i j}\right)\right\rangle$. Moreover, as readily expected, the second moment equations will contain the third moments, and so on. Thus, in order to obtain a complete set of equations, the $n$-th moment equations are necessary.

Let $\mathbf{p}$ be a vector with components $\left(p_{1}, \ldots, p_{N}\right)$ where $p_{i}$ is a non-negative integer. Furthermore, we introduce a function of $n_{i}$ given by

$$
\mathcal{M}(\mathbf{p})=\prod_{m=1}^{N} n_{m}^{\left(p_{m}\right)}
$$

where

$$
n_{m}^{\left(p_{m}\right)}=\prod_{i=0}^{p_{m}-1}\left(n_{m}-i\right)
$$

Multiplying Eq. (16) by $\mathcal{M}(\mathbf{p})$ and taking a sum over $\mathbf{n}$, we can obtain the equation of the factorial moment $\langle\mathcal{M}(\mathbf{p})\rangle$ :

$$
\begin{aligned}
\frac{\partial}{\partial t}\langle\mathcal{M}(\mathbf{p})\rangle & =\frac{\partial}{\partial t} \sum_{\mathbf{n}} \mathcal{M}(\mathbf{p}) f(\mathbf{n} ; t) \\
& =\frac{1}{2} \sum_{i, j=1}^{N} A_{i j} \sum_{\mathbf{n}} \mathcal{M}(\mathbf{p})\left[a_{i+j} a_{i}^{\dagger} n_{i} a_{j}^{\dagger} n_{j}-n_{i} a_{i} n_{j} a_{i}^{\dagger}\right] f(\mathbf{n} ; t) .
\end{aligned}
$$


Noting Eq. (17) and the commutation rules between the function $\mathcal{M}(\mathbf{p})$ and the operators $a_{i}, a_{i}^{\dagger}$

$$
\left\{\begin{array}{l}
\mathcal{M}(\mathbf{p}) a_{i}=a_{i} \mathcal{M}(\mathbf{p}) \frac{n_{i}+1}{n_{i}-p_{i}+1} \\
\mathcal{M}(\mathbf{p}) a_{i}^{\dagger}=a_{i}^{\dagger} \mathcal{M}(\mathbf{p}) \frac{n_{i}-p_{i}}{n_{i}}
\end{array}\right.
$$

we can eliminate $a_{i}$ and $a_{i}^{\dagger}$ from the above equation (cf. Eq. (23)) and the right-hand side of Eq. (28) becomes:

$$
\begin{aligned}
\frac{1}{2} \sum_{i, j=1}^{N} A_{i j}[ & p_{i+j}\left\langle\mathcal{M}(\mathbf{p}) \frac{\left(n_{i}-p_{i}\right)\left(n_{j}-p_{j}-\delta_{i j}\right)}{n_{i+j}-p_{i+j}+1}\right\rangle \\
& \left.\quad-2 p_{j}\left\langle\mathcal{M}(\mathbf{p})\left(n_{i}-p_{i}\right)\right\rangle-p_{i}\left(p_{j}-\delta_{i j}\right)\langle\mathcal{M}(\mathbf{p})\rangle\right] .
\end{aligned}
$$

In order to express this factorial moment equation in a simple form, we introduce again new operators $b_{i}$ and $b_{i}^{\dagger}$ which operate a vector $\mathbf{p}$ :

$$
\left\{\begin{array}{l}
b_{i} \mathcal{M}(\mathbf{p})=\mathcal{M}\left(\ldots, p_{i-1}, p_{i}-1, p_{i+1}, \ldots\right) \\
b_{i}^{\dagger} \mathcal{M}(\mathbf{p})=\mathcal{M}\left(\ldots, p_{i-1}, p_{i}+1, p_{i+1}, \ldots\right)
\end{array}\right.
$$

Using them, we finally have for the factorial moment equation

$$
\frac{\partial}{\partial t}\langle\mathcal{M}(\mathbf{p})\rangle=\frac{1}{2} \sum_{i, j=1}^{N} A_{i j}\left[p_{i+j}\left\langle b_{i+j} b_{i}^{\dagger} b_{j}^{\dagger} \mathcal{M}(\mathbf{p})\right\rangle-2 p_{j}\left\langle b_{i}^{\dagger} \mathcal{M}(\mathbf{p})\right\rangle-p_{i}\left(p_{j}-\delta_{i j}\right)\langle\mathcal{M}(\mathbf{p})\rangle\right] .
$$

\section{Solutions of the Stochastic Coagulation Equation for the Bilinear Coalescence Rates}

In the previous section, we derived the stochastic coagulation equation, which gives the probability function $f(\mathbf{n} ; t)$, and the factorial moment of this equation. Unfortunately, for the general case, solving these equations presents almost insurmountable difficulties. However, we can obtain the factorial moment $\langle\mathcal{M}(\mathbf{p})\rangle$ analytically, at least, for the special coalescence rates given by a bilinear form of masses of colliding particles (i.e., $A_{i j}=1, i+j$, and $i \times j$ ). Here, we introduce the solution to the stochastic coagulation equation for these coalescence rates.

\subsection{The case $A_{i j}=1$ and $i+j$}

When $A_{i j}=1$ and $i+j$, information about the total number of particles can readily be obtained. Thus, to begin with, we consider the total number of particles and its probability function. Using components of a state vector, the total particle number $\bar{N}$ in the state $\mathbf{n}$ is given by

$$
\bar{N}=\sum_{i=1}^{N} n_{i}
$$

Let $F\left(\bar{N}_{0} ; t\right)$ be the probability that $\bar{N}_{0}$-particles exist at time $t$. By taking a sum of the probability functions $f(\mathbf{n} ; t)$ over all possible states in which the total particle number is equal to $\bar{N}_{0}$, we have for $F\left(\bar{N}_{0} ; t\right)$

$$
F\left(\bar{N}_{0} ; t\right)=\mathcal{L}_{\bar{N}_{0}} f(\mathbf{n} ; t)
$$


where the operator $\mathcal{L}_{\bar{N}_{0}}$ is defined by

$$
\mathcal{L}_{\bar{N}_{0}}=\sum_{\mathbf{n}} \delta_{\bar{N} \bar{N}_{0}}
$$

When $A_{i j}=1$ and $i+j, F\left(\bar{N}_{0} ; t\right)$, which will be analytically obtained here, plays an important role in solving the stochastic coagulation equation.

For later convenience, we rewrite the stochastic coagulation equation into the following form: transposing the second term on the right side of Eq. (16) to the left and using Eq. (15), we obtain

$$
\left\{\frac{\partial}{\partial t}+\frac{1}{2} \sum_{i, j=1}^{N} A_{i j} n_{i}\left(n_{j}-\delta_{i j}\right)\right\} f(\mathbf{n} ; t)=\frac{1}{2} \sum_{i, j=1}^{N} A_{i j} a_{i+j} a_{i}^{\dagger} a_{j}^{\dagger} n_{i}\left(n_{j}-\delta_{i j}\right) f(\mathbf{n} ; t) .
$$

Furthermore, we introduce a function $\alpha$ given by

$$
\alpha=\frac{1}{2} \sum_{i, j=1}^{N} A_{i j} n_{i}\left(n_{j}-\delta_{i j}\right)
$$

$\alpha$ means the decay rate of the state $\mathbf{n}$. Though $\alpha$ is a function of the state vector $\mathbf{n}$ generally, $\alpha$ is expressed as a function of the total particle number $\bar{N}$ for $A_{i j}=1$ and $i+j$. That is, $\alpha$ is written by ${ }^{1}$

$$
\alpha= \begin{cases}\bar{N}(\bar{N}-1) / 2, & \text { for } A_{i j}=1 \\ N(\bar{N}-1), & \text { for } A_{i j}=i+j\end{cases}
$$

In order to emphasize that, for the coalescence rates $A_{i j}=1$ and $i+j, \alpha$ is a function of the total particle number $\bar{N}, \alpha$ is here referred to as $\alpha(\bar{N})$. This property of $\alpha(\bar{N})$ is essential for further analysis of the cases $A_{i j}=1$ and $i+j$. Using $\alpha(\bar{N})$, the stochastic coagulation equation is finally written in the following form:

$$
\left\{\frac{\partial}{\partial t}+\alpha(\bar{N})\right\} f(\mathbf{n} ; t)=\frac{1}{2} \sum_{i, j=1}^{N} A_{i j} a_{i+j} a_{i}^{\dagger} a_{j}^{\dagger} n_{i}\left(n_{j}-\delta_{i j}\right) f(\mathbf{n} ; t) .
$$

To obtain the equation for $F\left(\bar{N}_{0} ; t\right)$ we apply the operator $\mathcal{L}_{\bar{N}_{0}}$ to Eq. $(39)$; the left-hand side of Eq. (39) becomes

$$
\mathcal{L}_{\bar{N}_{0}}\left\{\frac{\partial}{\partial t}+\alpha(\bar{N})\right\} f(\mathbf{n} ; t)=\left\{\frac{\partial}{\partial t}+\alpha\left(\bar{N}_{0}\right)\right\} F\left(\bar{N}_{0} ; t\right) .
$$

In the above, we used Eq. (35) together with the following equation

$$
\sum_{\mathbf{n}} \delta_{\bar{N} \bar{N}_{0}} S(\bar{N}) T(\mathbf{n})=S\left(\bar{N}_{0}\right) \sum_{\mathbf{n}} \delta_{\bar{N}} \bar{N}_{0} T(\mathbf{n}),
$$

where $S(\bar{N})$ and $T(\mathbf{n})$ are functions of the total particle number $\bar{N}$ and that of the state vector $\mathbf{n}$, respectively. Using Eq. (41) and the commutation rules

$$
\left\{\begin{array}{l}
\delta_{\bar{N} \bar{N}_{0}} a_{i}=a_{i} \delta_{\bar{N}, \bar{N}_{0}-1} \\
\delta_{\bar{N} \bar{N}_{0}} a_{i}^{\dagger}=a_{i}^{\dagger} \delta_{\bar{N}, \bar{N}_{0}+1}
\end{array}\right.
$$

\footnotetext{
${ }^{1}$ In the case of $A_{i j}=i \times j, \alpha$ is given by $N^{2} \sum_{i} i^{2} n_{i}$ and, in fact, depends explicitly on a state vector $\mathbf{n}$.
} 
the right-hand side of Eq. (39) can be transformed as follows:

$$
\begin{aligned}
\mathcal{L}_{\bar{N}_{0}} \frac{1}{2} \sum_{i, j=1}^{N} A_{i j} a_{i+j} a_{i}^{\dagger} a_{j}^{\dagger} & n_{i}\left(n_{j}-\delta_{i j}\right) f(\mathbf{n} ; t) \\
& =\frac{1}{2} \sum_{i, j=1}^{N} A_{i j} \sum_{\mathbf{n}} \delta_{\bar{N}, \bar{N}_{0}+1} n_{i}\left(n_{j}-\delta_{i j}\right) f(\mathbf{n} ; t) \\
& =\alpha\left(\bar{N}_{0}+1\right) F\left(\bar{N}_{0}+1 ; t\right) .
\end{aligned}
$$

Accordingly, we have for the time-development equation of $F\left(\vec{N}_{0} ; t\right)$

$$
\left\{\frac{\partial}{\partial t}+\alpha\left(\bar{N}_{0}\right)\right\} F\left(\bar{N}_{0} ; t\right)=\alpha\left(\bar{N}_{0}+1\right) F\left(\bar{N}_{0}+1 ; t\right) .
$$

Now we will solve Eq. (44). As an initial condition, we take a simple and natural one: initially, $N$ particles exist and all particles have the same mass which is put to be unity. Then, the probability function of the total particle number is given, at $t=0$, by

$$
F(\bar{N} ; 0)=\delta_{\bar{N} N}
$$

Under the above initial condition, Eq. (44) can be solved as follows:

$$
F(N ; t)=\mathrm{e}^{-\alpha(N) t}
$$

and

$$
F\left(\bar{N}_{0} ; t\right)=\left\{\prod_{\bar{N}=\bar{N}_{0}+1}^{N} \alpha(\bar{N})\right\} \sum_{i=\bar{N}_{0}}^{N}\left\{\mathrm{e}^{-\alpha(i) t} / \prod_{\substack{j=\bar{N}_{0} \\ j \neq i \\ \text { for } \bar{N}_{0}<N .}}^{N}(\alpha(j)-\alpha(i))\right\},
$$

When $A_{i j}=1$ and $i+j$, using Eq. (38) we obtain

$$
\begin{gathered}
F\left(\bar{N}_{0} ; t\right)=\frac{N !(N-1) !}{\bar{N}_{0} !\left(\bar{N}_{0}-1\right) !} \sum_{\bar{N}=\bar{N}_{0}}^{N}(-1)^{\bar{N}-\bar{N}_{0}} \frac{(2 \bar{N}-1)\left(\bar{N}+\bar{N}_{0}-2\right) !}{(N-\bar{N}) !\left(\bar{N}-\bar{N}_{0}\right) !(N+\bar{N}-1) !} \mathrm{e}^{-\frac{\bar{N}(\bar{N}-1)}{2} t}, \\
\quad \text { for } A_{i j}=1
\end{gathered}
$$

and

$$
F\left(\bar{N}_{0} ; t\right)={ }_{N-1} \mathrm{C}_{N-\bar{N}_{0}} \sum_{\bar{N}=\bar{N}_{0}}^{N}(-1)^{\bar{N}-\bar{N}_{0}}{ }_{N-\bar{N}_{0}} \mathrm{C}_{N-\bar{N}} \mathrm{e}^{-(\bar{N}-1) N t}
$$

$$
\text { for } A_{i j}=i+j \text {, }
$$

where ${ }_{n} \mathrm{C}_{r}$ is the binomial coefficient. By using the probability function for the total particle number $F\left(\bar{N}_{0} ; t\right)$, the expectation of the total particle number $\langle\bar{N}\rangle_{N}$ is expressed by

$$
\langle\bar{N}\rangle_{N}=\sum_{\bar{N}=1}^{N} \bar{N} F(\bar{N} ; t)
$$

Thus, in the case of $A_{i j}=1$ we obtain

$$
\langle\bar{N}\rangle_{N}=\sum_{l=1}^{N}(2 l-1) \frac{N !(N-1) !}{(N-l) !(N+l-1) !} \mathrm{e}^{-\frac{l(l-1)}{2} t} \quad\left(A_{i j}=1\right)
$$


and in the case of $A_{i j}=i+j$

$$
\langle\bar{N}\rangle_{N}=1+(N-1) \mathrm{e}^{-\tau} \quad\left(A_{i j}=i+j\right),
$$

where

$$
\tau=N t
$$

When $A_{i j}=i+j$, as seen from Eq. (51), in the limit $N \rightarrow \infty\langle\bar{N}\rangle_{N}$ agrees with the total particle number given by the ordinary coagulation equation, $\sum N_{i}(t)=N \mathrm{e}^{-\tau}$. On the other hand, for $A_{i j}=1$, we cannot tell at a glance whether $\langle\vec{N}\rangle_{N}$ agrees with the total particle number $\sum N_{i}(t)=N /(1+\tau / 2)$ given by the coagulation equation in the limit of $N \rightarrow \infty$ because Eq. (50) is expressed by the complex sum. However, in actual fact, by seeing the change of $\langle\bar{N}\rangle_{N} / N$ with the increase in $N$ (in Fig. 2), we know that, in the limit of $N \rightarrow \infty,\langle\bar{N}\rangle_{N}$ agrees with $\sum N_{i}(t)$ in this case, too.

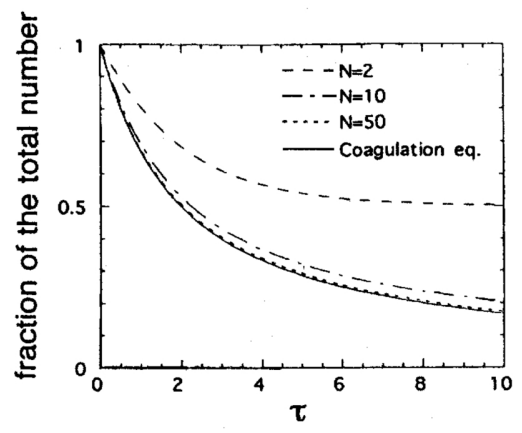

Fig. 2. The time-variation of the total number of particle $\langle\bar{N}\rangle$ for the case of $A_{i j}=1$. In the limit of the infinite initial total number, $\langle\tilde{N}\rangle$ agrees with that given by the ordinary coagulation equation.

Before seeking the moment $\langle\mathcal{M}(\mathbf{p})\rangle$, we will mention another property for the case of $A_{i j}=1$ and $i+j$. Using $F(\bar{N} ; t)$, we can write the state probability function as

$$
f(\mathbf{n} ; t)=F(\bar{N} ; t) g_{\bar{N} l},
$$

where each state with the total particle number $\bar{N}$ is distinguished by a label $l$. As seen from its definition, $g_{\bar{N} l}$ is the probability occupied by the state $(\bar{N}, l)$ in all of possible states with the total particle number $\bar{N}$. Then, taking a sum of Eq. (53) over $l$ and noting Eq. (34), we have

$$
\sum_{l} g_{\bar{N} l}=1
$$

When $A_{i j}=1$ and $i+j, g_{\bar{N} l}$ can be proved to be independent of time $t$ (this proof will be presented in the Appendix).

Now, we seek the factorial moment $\langle\mathcal{M}(\mathbf{p})\rangle$. Generally, the factorial moment $\langle\mathcal{M}(\mathbf{p})\rangle$ depends on the total mass $N$. To express this explicitly, we will denote the factorial moment $\langle\mathcal{M}(\mathbf{p})\rangle$ by $\langle\mathcal{M}(\mathbf{p})\rangle_{N}$ hereafter. By the use of $F(\bar{N} ; t)$ and $g_{\bar{N} l}$, we can rewrite $\langle\mathcal{M}(\mathbf{p})\rangle_{N}$ as follows:

$$
\langle\mathcal{M}(\mathbf{p})\rangle_{N}=\sum_{\mathbf{n}} \mathcal{M}(\mathbf{p}) F(\bar{N} ; t) g_{\bar{N} l}
$$




$$
\begin{aligned}
& =\sum_{\bar{N}_{0}=1}^{N} F\left(\bar{N}_{0} ; t\right) \mathcal{L}_{\bar{N}_{0}} \mathcal{M}(\mathbf{p}) g_{\bar{N} l} \\
& =\sum_{\bar{N}_{0}=1}^{N} F\left(\bar{N}_{0} ; t\right)\langle\langle\mathcal{M}(\mathbf{p})\rangle\rangle_{\left(N, \bar{N}_{0}\right)},
\end{aligned}
$$

where

$$
\left\langle\langle\mathcal{M}(\mathbf{p})\rangle_{\left(N, \bar{N}_{0}\right)}=\mathcal{L}_{\bar{N}_{0}} \mathcal{M}(\mathbf{p}) g_{\bar{N} l} .\right.
$$

The above quantity $\left\langle\langle\mathcal{M}(\mathbf{p})\rangle_{\left(N, \bar{N}_{0}\right)}\right.$ is the expectation value of $\mathcal{M}(\mathbf{n})$ averaged over the states with the total particle number $\bar{N}_{0}$ and is independent of $t$ as well as $g_{\bar{N} l}$.

To obtain the factorial moment $\langle\mathcal{M}(\mathbf{p})\rangle_{N}$, we will first consider $\left\langle\langle\mathcal{M}(\mathbf{p})\rangle_{\left(N, \bar{N}_{0}\right)}\right.$. When the total particle number is equal to the total mass $N$, all particles must have the unit mass. Thus, $\left\langle\langle\mathcal{M}(\mathbf{p})\rangle_{(N, N)}\right.$ is given by

$$
\begin{cases}\left\langle\left\langle\mathcal{M}\left(\mathbf{p}_{\mathbf{1}}\right)\right)\right\rangle_{(N, N)}={ }_{N} \mathrm{P}_{p_{1}}, & \text { for } \mathbf{p}_{1}=\left(p_{1}, 0, \ldots, 0\right), \quad 0 \leq p_{1} \leq N \\ \langle\langle\mathcal{M}(\mathbf{p})\rangle\rangle_{(N, N)}=0, & \text { for } \mathbf{p} \neq \mathbf{p}_{1},\end{cases}
$$

where $0 \leq p_{1} \leq N$ and ${ }_{N} \mathrm{P}_{p_{1}}$ is the number of permutation. For further investigation of $\left\langle\langle\mathcal{M}(\mathbf{p})\rangle_{\left(N, \bar{N}_{0}\right)}\right.$, let us derive the equation giving $\langle\langle\mathcal{M}(\mathbf{p})\rangle\rangle_{\left(N, \bar{N}_{0}\right)}$ form $\left\langle\langle\mathcal{M}(\mathbf{p})\rangle_{(N, N)}\right.$. We multiply Eq. (39) by $\mathcal{M}(\mathbf{p})$ and apply the operator $\mathcal{L}_{\bar{N}_{0}}$ to it. Noting that $g_{\bar{N} l}$ is independent of time $t$ and using Eqs. (44) and (56), we can transform the left-hand side of Eq. (39) as follows:

$$
\begin{aligned}
\mathcal{L}_{\bar{N}_{0}} \mathcal{M}(\mathbf{p})\left\{\frac{\partial}{\partial t}+\alpha(\bar{N})\right\} f(\mathbf{n} ; t) & =\left\{\frac{\partial}{\partial t}+\alpha\left(\bar{N}_{0}\right)\right\} F\left(\bar{N}_{0} ; t\right) \mathcal{L}_{\bar{N}_{0}} \mathcal{M}(\mathbf{p}) g_{\bar{N} l} \\
& =\alpha\left(\bar{N}_{0}+1\right) F\left(\bar{N}_{0}+1 ; t\right)\langle\langle\mathcal{M}(\mathbf{p})\rangle\rangle_{\left(N, \bar{N}_{0}\right)} .
\end{aligned}
$$

The right-hand side is transformed in the same way that was performed in deriving the factorial moment equation itself:

$$
\begin{aligned}
& \mathcal{L}_{\bar{N}_{0}} \mathcal{M}(\mathbf{p}) \frac{1}{2} \sum_{i, j=1}^{N} A_{i j} a_{i+j} a_{i}^{\dagger} a_{j}^{\dagger} n_{i}\left(n_{j}-\delta_{i j}\right) f(\mathbf{n} ; t) \\
& =F\left(\bar{N}_{0}+1 ; t\right) \sum_{\mathbf{n}} \delta_{\bar{N} \bar{N}_{0}+1} \\
& \quad \times \sum_{i, j=1}^{N} \frac{1}{2} A_{i j}\left\{\left(n_{i}-p_{i}\right)\left(n_{j}-p_{j}-\delta_{i j}\right) \mathcal{M}(\mathbf{p})+p_{i+j}\left(b_{i+j} b_{i}^{\dagger} b_{j}^{\dagger} \mathcal{M}(\mathbf{p})\right)\right\} g_{\bar{N} l} .
\end{aligned}
$$

Furthermore, we introduce a function $\beta$ defined by

$$
\beta=\sum_{i, j=1}^{N} \frac{1}{2} A_{i j}\left(n_{i}-p_{i}\right)\left(n_{j}-p_{j}-\delta_{i j}\right)
$$

In the cases of $A_{i j}=1$ and $i+j, \beta$, as well as $\alpha(\bar{N})$, is a function of the total particle number rather than a state vector:

$$
\beta= \begin{cases}(\bar{N}-\bar{P})(\bar{N}-\bar{P}-1) / 2, & \text { for } A_{i j}=1 \\ (N-P)(\bar{N}-\bar{P}-1), & \text { for } A_{i j}=i+j\end{cases}
$$


where

$$
\bar{P}=\sum_{i=1}^{N} p_{i} \quad \text { and } \quad P=\sum_{i=1}^{N} i p_{i} .
$$

Using $\beta(\bar{N})$, we have for the right-hand side of Eq. (59)

$$
\begin{aligned}
& F\left(\bar{N}_{0}+1 ; t\right)\left[\beta\left(\bar{N}_{0}+1\right)\langle\langle\mathcal{M}(\mathbf{p})\rangle\rangle_{\left(N, \bar{N}_{0}+1\right)}\right. \\
& \left.\quad+\sum_{i, j=1}^{N} \frac{1}{2} p_{i+j} A_{i j}\left\langle\left\langle b_{i+j} b_{i}^{\dagger} b_{j}^{\dagger} \mathcal{M}(\mathbf{p})\right\rangle\right\rangle_{\left(N, \bar{N}_{0}+1\right)}\right] .
\end{aligned}
$$

By the use of Eqs. (58) and (63), the equation for $\langle\langle\mathcal{M}(\mathbf{p})\rangle\rangle_{\left(N, \bar{N}_{0}\right)}$ is obtained as

$$
\begin{aligned}
\alpha\left(\bar{N}_{0}+1\right)\langle\langle\mathcal{M}(\mathbf{p})\rangle\rangle_{\left(N, \bar{N}_{0}\right)}= & \beta\left(\bar{N}_{0}+1\right)\langle\langle\mathcal{M}(\mathbf{p})\rangle\rangle_{\left(N, \bar{N}_{0}+1\right)} \\
& +\sum_{k=1}^{N} \sum_{i, j=1}^{N} \delta_{i+j, k} \frac{1}{2} p_{k} A_{i j}\left\langle\left\langle b_{k} b_{i}^{\dagger} b_{j}^{\dagger} \mathcal{M}(\mathbf{p})\right\rangle_{\left(N, \bar{N}_{0}+1\right)} .\right.
\end{aligned}
$$

We can find the solution to Eq. (64), using Eq. (57). That is, in the case of $A_{i j}=1$ we have for $\langle\langle\mathcal{M}(\mathbf{p})\rangle\rangle_{\left(N, \bar{N}_{0}\right)}$

$$
\begin{array}{r}
\langle\langle\mathcal{M}(\mathbf{p}))\rangle_{\left(N, \bar{N}_{0}\right)}={ }_{N-\bar{N}_{0}} \mathrm{P}_{P-\bar{P}}\left\{\prod_{i=1}^{\bar{P}}\left(\bar{N}_{0}-i+1\right)\left(\bar{N}_{0}-i\right)\right\} /\left\{\prod_{i=1}^{P}(N-i)\right\} \\
\left(A_{i j}=1\right),
\end{array}
$$

and in the case of $A_{i j}=i+j$

$$
\begin{aligned}
& \left\langle\langle\mathcal{M}(\mathbf{p})\rangle_{\left(N, \bar{N}_{0}\right)}={ }_{N-\bar{N}_{0}} \mathrm{P}_{P-\bar{P}} \frac{(N-P)^{N-\bar{N}-P+\bar{P}-1}}{N^{N-\bar{N}-1}}\left\{\prod_{k=1}^{N}\left(\frac{k^{k-1}}{k !}\right)^{p_{k}}\right\}\left\{\prod_{i=1}^{\bar{P}}(\bar{N}-i)\right\}\right. \\
& \left(A_{i j}=i+j\right) \text {. }
\end{aligned}
$$

By the direct substitution, we can confirm that these solutions satisfy Eq. (64).

To obtain our requiring quantity $\langle\mathcal{M}(\mathbf{p})\rangle_{N}$, we now substitute the expressions of $F\left(\bar{N}_{0} ; t\right)$ and $\left\langle\langle\mathcal{M}(\mathbf{p})\rangle_{\left(N, \bar{N}_{0}\right)}\right.$ (i.e., Eqs. (47) and (65) for $A_{i j}=1$, or Eqs. (48) and (66) for $A_{i j}=i+j$ ) into Eq. (55). In the case of where $A_{i j}=1$, we have for $\langle\mathcal{M}(\mathbf{p})\rangle_{N}$

$$
\begin{aligned}
&\langle\mathcal{M}(\mathbf{p})\rangle_{N}={ }_{N} \mathrm{P}_{P} \sum_{\bar{N}_{0}=\bar{P}}^{N-P+\bar{P}} \sum_{\bar{N}=\bar{N}_{0}}^{N}(-1)^{\bar{N}-\bar{N}_{0}} \frac{(2 \bar{N}-1){ }_{N-1} \mathrm{C}_{\bar{N}_{0}-1 N-P} \mathrm{C}_{\bar{N}_{0}-\bar{P} \quad N-\bar{N}_{0}} \mathrm{C}_{N-\bar{N}}}{\left(\bar{N}+\bar{N}_{0}-1\right)_{N+\bar{N}-1} \mathrm{C}_{\bar{N}+\bar{N}_{0}-1}} \times\left\{\prod_{i=1}^{\bar{P}}\left(\bar{N}_{0}-i\right) / \prod_{i=1}^{P}(N-i)\right\} \mathrm{e}^{-\frac{\bar{N}(\bar{N}-1)}{2} t} \\
&\left(A_{i j}=1\right) .
\end{aligned}
$$

On the other hand, in the case of where $A_{i j}=i+j,\langle\mathcal{M}(\mathbf{p})\rangle_{N}$ can be expressed in a simple form:

$$
\begin{aligned}
&\langle\mathcal{M}(\mathbf{p})\rangle_{N}=\frac{{ }_{N} \mathrm{P}_{P}}{N^{P-\bar{P}}}\left\{\prod_{k=1}^{N}\left(\frac{k^{k-1}}{k !}\right)^{p_{k}}\right\}\left\{1-\frac{P}{N}\left(1-\mathrm{e}^{-\tau}\right)\right\}^{N-P-1} \\
& \times\left(1-\mathrm{e}^{-\tau}\right)^{P-\bar{P}} \mathrm{e}^{-\bar{P} \tau} \quad\left(A_{i j}=i+j\right),
\end{aligned}
$$


where

$$
\tau=N t .
$$

Finally, for the case of $A_{i j}=i+j$, by using the above result, we will discuss the condition that the moment $\left\langle n_{k}\right\rangle_{N}$ agrees approximately with the solution to the ordinary coagulation equation $N_{k}$. From Eq. (68), $\left\langle n_{k}\right\rangle_{N}$ becomes

$$
\left\langle n_{k}\right\rangle_{N}=\frac{{ }_{N} \mathrm{P}_{k}}{N^{k-1}} \frac{k^{k-1}}{k !}\left\{1-\frac{k}{N}\left(1-\mathrm{e}^{-\tau}\right)\right\}^{N-k-1}\left(1-\mathrm{e}^{-\tau}\right)^{k-1} \mathrm{e}^{-\tau}
$$

Here, we consider the particles which have small masses $k(\ll N)$. Then, we can rewrite

$$
{ }_{N} \mathrm{P}_{k}=N^{k} \mathrm{e}^{-k^{2} / 2 N}
$$

and

$$
\left\{1-\frac{k}{N}\left(1-\mathrm{e}^{-\tau}\right)\right\}^{N-k-1}=\mathrm{e}^{-k\left(1-\mathrm{e}^{-\tau}\right)} \mathrm{e}^{\left(1-\mathrm{e}^{-2 \tau}\right) k^{2} / 2 N}
$$

within a good accuracy. Substituting these equations into Eq. (70), we have for $\left\langle n_{k}\right\rangle_{N}$

$$
\left\langle n_{k}\right\rangle_{N}=N \frac{k^{k-1}}{k !} \frac{\mathrm{e}^{-\tau}}{1-\mathrm{e}^{-\tau}} \mathrm{e}^{-k\left[\left(1-\mathrm{e}^{-\tau}\right)-\ln \left(1-\mathrm{e}^{-\tau}\right)\right]} \times \mathrm{e}^{-\frac{k^{2}}{2 N} \mathrm{e}^{-2 \tau}} .
$$

The above expression of $\left\langle n_{k}\right\rangle_{N}$ is the same as that of the ordinary coagulation equation $N_{k}$ obtained by Trubnikov (1971), except for a factor $\mathrm{e}^{-\frac{k^{2}}{2 N} \mathrm{e}^{-2 \tau}}$. Consequently, we can conclude that, for the case of $A_{i j}=i+j,\left\langle n_{k}\right\rangle_{N}$ always agrees with $N_{k}$ if $k^{2}$ is much smaller than $N$ (i.e., if $k$ is smaller than the geometrical mean of the smallest mass "1" and the total mass).

\subsection{The case $A_{i j}=i \times j$}

In the case of $A_{i j}=i \times j$, the factorial moments have a useful property which reduces the factorial moment equations to the first moment equation. Here, we will show the property of the factorial moments.

Now, we assume that at the initial time $t_{0}$ the factorial moments satisfy the following relation for a set of arbitrary vectors $\mathbf{p}$ and $\mathbf{r}:{ }^{2}$

$$
\langle\mathcal{M}(\mathbf{p}+\mathbf{r})\rangle_{N}=\langle\mathcal{M}(\mathbf{p})\rangle_{N}\langle\mathcal{M}(\mathbf{r})\rangle_{N-P}
$$

where

$$
P=\sum_{i=1}^{N} i p_{i} .
$$

Then, we can prove that Eq. (74) holds at any time. First, we will show that the time derivative of the right-hand side of Eq. (74) is equal to that of the left at time $t_{0}$. By the use of Eq. (32), we have for the time derivative of the right-hand side of Eq. (74)

$$
\begin{aligned}
& \frac{\partial}{\partial t}\langle\mathcal{M}(\mathbf{p})\rangle_{N}\langle\mathcal{M}(\mathbf{r})\rangle_{N-P} \\
& \quad=\frac{\partial\langle\mathcal{M}(\mathbf{p})\rangle_{N}}{\partial t}\langle\mathcal{M}(\mathbf{r})\rangle_{N-P}+\langle\mathcal{M}(\mathbf{p})\rangle_{N} \frac{\partial\langle\mathcal{M}(\mathbf{r})\rangle_{N-P}}{\partial t}
\end{aligned}
$$

\footnotetext{
${ }^{2}$ For the initial condition we adopt, as will be seen below, the equation (73) always holds. Thus, this assumption is the reasonable one.
} 


$$
\begin{aligned}
& =\sum_{k=1}^{N}\left[\sum _ { i , j = 1 } ^ { N } \frac { 1 } { 2 } A _ { i j } \delta _ { i + j , k } \left\{p_{k}\left\langle b_{k} b_{i}^{\dagger} b_{j}^{\dagger} \mathcal{M}(\mathbf{p})\right\rangle_{N}\langle\mathcal{M}(\mathbf{r})\rangle_{N-P}\right.\right. \\
& \left.\quad+r_{k}\langle\mathcal{M}(\mathbf{p})\rangle_{N}\left\langle b_{k} b_{i}^{\dagger} b_{j}^{\dagger} \mathcal{M}(\mathbf{r})\right\rangle_{N-P}\right\} \\
& \left.\quad-\sum_{i=1}^{N} A_{i k}\left\{p_{k}\left\langle b_{i} \mathcal{M}(\mathbf{p})\right\rangle_{N}\langle\mathcal{M}(\mathbf{q})\rangle_{N-P}+r_{k}\langle\mathcal{M}(\mathbf{p})\rangle_{N}\left\langle b_{i} \mathcal{M}(\mathbf{r})\right\rangle_{N-P}\right\}\right] \\
& -\sum_{i, j=1}^{N} A_{i j}\left\{\frac{p_{i}\left(p_{j}-\delta_{i j}\right)}{2}+\frac{r_{i}\left(r_{j}-\delta_{i j}\right)}{2}\right\}\langle\mathcal{M}(\mathbf{p})\rangle_{N}\langle\mathcal{M}(\mathbf{r})\rangle_{N-P} .
\end{aligned}
$$

Since $A_{i j}=i \times j$ in the present case, the right-hand side of Eq. (76) can be rewritten as

$$
\begin{gathered}
\sum_{k=1}^{N}\left[\sum _ { i , j = 1 } ^ { N } \frac { 1 } { 2 } i j \delta _ { i + j , k } \left\{p_{k}\left\langle b_{k} b_{i}^{\dagger} b_{j}^{\dagger} \mathcal{M}(\mathbf{p})\right\rangle_{N}\langle\mathcal{M}(\mathbf{r})\rangle_{N-P}\right.\right. \\
\left.+r_{k}\langle\mathcal{M}(\mathbf{p})\rangle_{N}\left\langle b_{k} b_{i}^{\dagger} b_{j}^{\dagger} \mathcal{M}(\mathbf{r})\right\rangle_{N-P}\right\} \\
-k\left\{p_{k}(N-P)\langle\mathcal{M}(\mathbf{p})\rangle_{N}\langle\mathcal{M}(\mathbf{r})\rangle_{N-P}\right. \\
\left.\left.+r_{k}\langle\mathcal{M}(\mathbf{p})\rangle_{N}((N-P)-R)\langle\mathcal{M}(\mathbf{r})\rangle_{N-P}\right\}\right] \\
-\sum_{i, j=1}^{N} i j\left\{\frac{p_{i}\left(p_{j}-\delta_{i j}\right)}{2}+\frac{r_{i}\left(r_{j}-\delta_{i j}\right)}{2}\right\}\langle\mathcal{M}(\mathbf{p})\rangle_{N}\langle\mathcal{M}(\mathbf{r})\rangle_{N-P}
\end{gathered}
$$

where

$$
R=\sum_{m=1}^{N} m r_{m}
$$

In the above, we used Eq. (10). Furthermore, from Eq. (74) we obtain the following equations at time $t_{0}$, i.e.,

$$
\left\{\begin{array}{l}
\langle\mathcal{M}(\mathbf{p})\rangle_{N}\langle\mathcal{M}(\mathbf{r})\rangle_{N-P}=\langle\mathcal{M}(\mathbf{p}+\mathbf{r})\rangle_{N}, \\
\left\langle b_{k} b_{i}^{\dagger} b_{j}^{\dagger} \mathcal{M}(\mathbf{p})\right\rangle_{N}\langle\mathcal{M}(\mathbf{r})\rangle_{N-P}=\left\langle b_{k} b_{i}^{\dagger} b_{j}^{\dagger} \mathcal{M}(\mathbf{p}+\mathbf{r})\right\rangle_{N}, \\
\langle\mathcal{M}(\mathbf{p})\rangle_{N}\left\langle b_{k} b_{i}^{\dagger} b_{j}^{\dagger} \mathcal{M}(\mathbf{r})\right\rangle_{N-P}=\left\langle b_{k} b_{i}^{\dagger} b_{j}^{\dagger} \mathcal{M}(\mathbf{p}+\mathbf{r})\right\rangle_{N} .
\end{array}\right.
$$

Substituting these equations into Eq. (77) and using Eq. (32) again, we have finally

$$
\begin{aligned}
& \frac{\partial}{\partial t}\langle\mathcal{M}(\mathbf{p})\rangle_{N}\langle\mathcal{M}(\mathbf{r})\rangle_{N-P} \\
& =\sum_{k=1}^{N}\left(p_{k}+r_{k}\right)\left[\sum_{i, j=1}^{N} \frac{1}{2} A_{i j} \delta_{i+j, k}\left\langle b_{k} b_{i}^{\dagger} b_{j}^{\dagger} \mathcal{M}(\mathbf{p}+\mathbf{r})\right\rangle_{N}\right. \\
& \left.\quad-k(N-(P+R))\langle\mathcal{M}(\mathbf{p}+\mathbf{r})\rangle_{N}\right] \\
& \quad-\left[\sum_{k=1}^{N} k p_{k} R+\sum_{i, j=1}^{N} A_{i j}\left\{\frac{p_{i}\left(p_{j}-\delta_{i j}\right)}{2}+\frac{r_{i}\left(r_{j}-\delta_{i j}\right)}{2}\right\}\right]\langle\mathcal{M}(\mathbf{p}+\mathbf{r})\rangle_{N} \\
& =\sum_{k=1}^{N}\left(p_{k}+r_{k}\right)\left[\sum_{i, j=1}^{N} \frac{1}{2} A_{i j} \delta_{i+j, k}\left\langle b_{k} b_{i}^{\dagger} b_{j}^{\dagger} \mathcal{M}(\mathbf{p}+\mathbf{r})\right\rangle_{N}\right.
\end{aligned}
$$




$$
\begin{gathered}
\left.-\sum_{i=1}^{N} A_{i k}\left\langle b_{i} \mathcal{M}(\mathbf{p}+\mathbf{r})\right\rangle_{N}\right] \\
-\sum_{i, j=1}^{N} A_{i j}\left\{\frac{\left(p_{i}+r_{i}\right)\left(p_{j}+r_{j}-\delta_{i j}\right)}{2}\right\}\langle\mathcal{M}(\mathbf{p}+\mathbf{r})\rangle_{N} \\
=\frac{\partial}{\partial t}\langle\mathcal{M}(\mathbf{p}+\mathbf{r})\rangle_{N} .
\end{gathered}
$$

That is, we see that the derivative of the left-hand side of Eq. (74) is equal to that of the right at time $t_{0}$.

In the same way, it is proved by recursion that we have the following equation at time $t_{0}$ :

$$
\frac{\partial^{n}}{\partial t^{n}}\langle\mathcal{M}(\mathbf{p}+\mathbf{r})\rangle_{N}=\frac{\partial^{n}}{\partial t^{n}}\left\{\langle\mathcal{M}(\mathbf{p})\rangle_{N}\langle\mathcal{M}(\mathbf{r})\rangle_{N-P}\right\}
$$

Thus, recalling the Taylor expansion theorem, we obtain the following result for the case of $A_{i j}=i \times j$ : if Eq. (74) holds at one time, it holds at any time.

Using this property of the factorial moments, we will now solve the factorial moment equations. As an initial condition, we adopt the same one that we took in Subsection 3.1. That is, there exist $N$ particles and all particles have the unit mass. Then, we have for the state probability function

$$
\left\{\begin{array}{l}
f\left(\mathbf{n}_{i} ; t=0\right)=1 \\
f(\mathbf{n} ; t=0)=0
\end{array} \text { for } \mathbf{n} \neq \mathbf{n}_{i}\right.
$$

where

$$
\mathbf{n}_{i}=(N, 0, \ldots, 0)
$$

By noting Eqs. (26) and (27), the factorial moments are given, at $t=0$, by

$$
\left\{\begin{array}{l}
\left\langle\mathcal{M}\left(\mathbf{p}_{\mathbf{1}}\right)\right\rangle_{N}={ }_{N} \mathrm{P}_{p_{1}}, \\
\langle\mathcal{M}(\mathbf{p})\rangle_{N}=0,
\end{array} \text { for } \mathbf{p} \neq \mathbf{p}_{\mathbf{1}},\right.
$$

where

$$
\mathbf{p}_{1}=\left(p_{1}, 0, \ldots, 0\right), \quad\left(0 \leq p_{1} \leq N\right) .
$$

It should be noticed that these values of the factorial moments satisfy Eq. (74). Hence, according to the property of the factorial moments mentioned in the previous subsection, we have for an arbitrary set of vectors $\mathbf{p}$ and $\mathbf{r}$

$$
\langle\mathcal{M}(\mathbf{p}+\mathbf{r})\rangle_{N}=\langle\mathcal{M}(\mathbf{p})\rangle_{N}\langle\mathcal{M}(\mathbf{r})\rangle_{N-P}, \quad \text { at any time. }
$$

Let us consider the time variation of $\left\langle n_{k}\right\rangle_{N}$. In the case of $A_{i j}=i \times j$ the first moment equation (24) becomes

$$
\frac{\partial}{\partial t}\left\langle n_{k}\right\rangle_{N}=\frac{1}{2} \sum_{i, j=1}^{N} i j \delta_{i+j, k}\left\langle n_{i}\left(n_{j}-\delta_{i j}\right)\right\rangle_{N}-\sum_{i=1}^{N} i k\left\langle n_{k}\left(n_{i}-\delta_{i k}\right)\right\rangle_{N}
$$

Furthermore, from Eq. (86) we obtain

$$
\left\{\begin{array}{l}
\left\langle n_{i}\left(n_{j}-\delta_{i j}\right)\right\rangle_{N}=\left\langle n_{i}\right\rangle_{N}\left\langle n_{j}\right\rangle_{N-i} \\
\left\langle n_{k}\left(n_{i}-\delta_{k i}\right)\right\rangle_{N}=\left\langle n_{k}\right\rangle_{N}\left\langle n_{i}\right\rangle_{N-k}
\end{array}\right.
$$


Substituting these equations into Eq. (87), we have for the first moment equation

$$
\frac{\partial}{\partial t}\left\langle n_{k}\right\rangle_{N}=\frac{1}{2} \sum_{i, j=1}^{N} i j \delta_{i+j, k}\left\langle n_{i}\right\rangle_{N}\left\langle n_{j}\right\rangle_{N-i}-k(N-k)\left\langle n_{k}\right\rangle_{N},
$$

where we used (see Eq. (10))

$$
\sum_{i=1}^{N}\left\langle i n_{i}\right\rangle_{N-k}=N-k
$$

The obtained equations are complete mathematically and, hence, we can find $\left\langle\mathcal{M}\left(\mathbf{n}_{\mathbf{k}}\right)\right\rangle_{N}$ without any information on the higher moments.

Noting a form of Eq. (89), we may presume that $\left\langle n_{k}\right\rangle_{N}$ can be written as

$$
\left\langle n_{k}\right\rangle_{N}={ }_{N} \mathrm{C}_{k} \mathrm{e}^{-k(N-k) t} f_{k}(t) .
$$

Substituting Eq. (91) into Eq. (89), we obtain the equation for $f_{k}(t)$ :

$$
\frac{\partial f_{k}(t)}{\partial t}=\frac{1}{2} \sum_{i, j=1}^{N} i j \delta_{i+j, k} \mathrm{C}_{i} \mathrm{e}^{-i j t} f_{i}(t) f_{j}(t) .
$$

Furthermore, from Eq. (84) we have for the initial conditions of $f_{k}$

$$
f_{i}(0)=\delta_{i 1} \text {. }
$$

Under the above initial conditions, we can find $f_{k}(t)$ from Eq. (92) successively:

$$
\begin{aligned}
& f_{1}=1, \\
& f_{2}=(1-x), \\
& f_{3}=(1-x)^{2}(1+2 x), \\
& f_{4}=(1-x)^{3}\left(1+3 x+6 x^{2}+6 x^{3}\right), \\
& f_{5}=(1-x)^{4}\left(1+4 x+10 x^{2}+20 x^{3}+30 x^{4}+36 x^{5}+24 x^{6}\right),
\end{aligned}
$$

where

$$
x=\mathrm{e}^{-t} .
$$

Although it is difficult to write down the general form of $f_{k}(x)$, we can readily show that the function $f_{k}(x)$ has the following form

$$
f_{k}(x)=(1-x)^{k-1} h_{k}(x) .
$$

In the above, $h_{k}(x)$ is a polynomial function of $x$ of the degree of $(k-1)(k-2) / 2$ :

$$
h_{k}(x)=\sum_{l=0}^{\frac{(k-1)(k-2)}{2}} D_{k l} x^{l}
$$

where the coefficients $D_{k l}$ are all integer and have the following properties, i.e.,

$$
\left\{\begin{array}{l}
D_{k 1}=1 \\
D_{k, \frac{(k-1)(k-2)}{2}}=(k-1) ! \\
\sum_{l} D_{k l}^{2}=k^{k-2}
\end{array}\right.
$$


Figure 3 shows the time-variation of $\left\langle n_{k}\right\rangle$ for the case of $A_{i j}=i \times j$ and $N=25$ given by Eqs. (91) and (92). For comparison, we add the solution of the "ordinary" coagulation equation (1) for the same coalescence rate and the same initial condition. We can see from this figure that, for the small masses $k=1$ and 4 , both solutions are closely coincident and that, for the large masses $k=16$ and 25 , two equations give quantitatively different expectation values. It is also seen that $\left\langle n_{25}\right\rangle$ approaches the value 1 as $t \rightarrow \infty$ for the stochastic coagulation equation, but vanishes for the ordinary coagulation equation. This means the serious defect of the ordinary coagulation equation that the total mass cannot be conserved in a system.

Finally, we will discuss the condition that the moment $\left\langle n_{k}\right\rangle_{N}$ agrees approximately with the solution to the ordinary coagulation equation $N_{k}$. We consider the particles having relatively small masses $k$ which satisfy

$$
k^{2} \ll N .
$$

Furthermore, we limit ourselves only to the early stage of coagulation process, that is,

$$
t \lesssim \frac{1}{N}
$$

Then, we can see that $k^{2} t$ is much smaller than the unity:

$$
k^{2} t \lesssim \frac{k^{2}}{N} \ll 1
$$

(a)

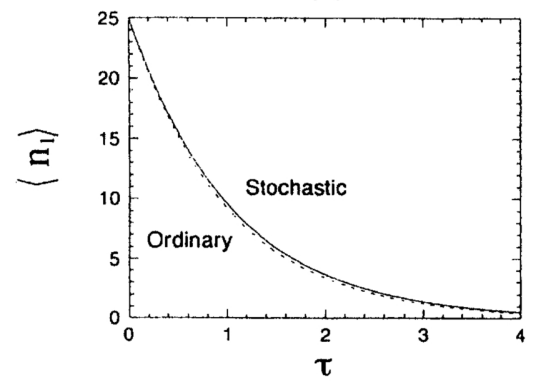

(c)

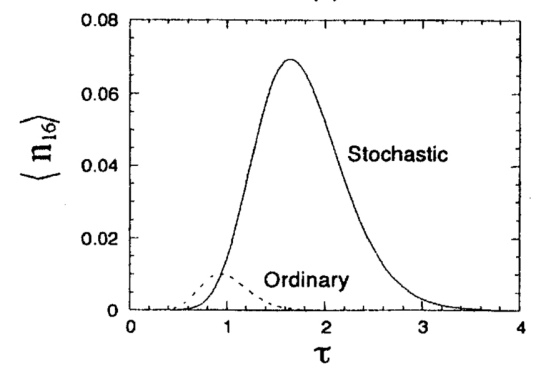

(b)

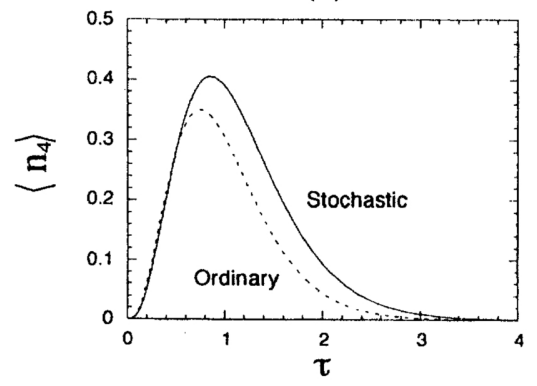

(d)

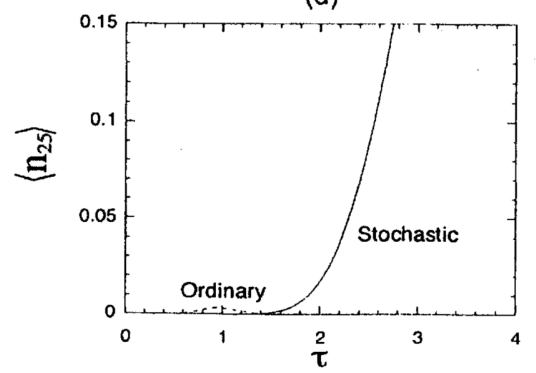

Fig. 3. Comparison of the solution to the stochastic coagulation equation (solid line) with that to the ordinary coagulation equation (dashed line) for the case of $A_{i j}=i \times j$ and $N=25$. The expectation values $\left\langle n_{k}\right\rangle$ are shown for the masses $k=1,4,16$, and 25 (in figures (a), (b), (c), and (d), respectively). For the small masses $k=1$ and 4 , both solutions are closely coincident but, for the large masses $k=16$ and 25 , two equations give quantitatively different values. 
In this case we can rewrite as

$$
\mathrm{e}^{-k^{2} t}=1
$$

and

$$
{ }_{N} \mathrm{C}_{k}=\frac{N^{k}}{k !}
$$

within a good accuracy. By the use of these equations, Eqs. (91) and (92) can be approximated, respectively, as

$$
\left\langle n_{k}\right\rangle_{N}=\frac{N^{k}}{k !} \mathrm{e}^{-k N t} f_{k}(t)
$$

and

$$
\frac{\partial f_{k}(t)}{\partial t}=\frac{1}{2} \sum_{i, j=1}^{N} i j \delta_{i+j, k k} \mathrm{C}_{i} f_{i}(t) f_{j}(t) .
$$

The solution to Eq. (105) can be found to be (Trubnikov, 1971)

$$
f_{k}(t)=k^{k-2} t^{k-1} \text {. }
$$

Substituting Eq. (106) into Eq. (104), we have for $\left\langle n_{k}\right\rangle_{N}$

$$
\left\langle n_{k}\right\rangle_{N}=N \frac{k^{k-2}}{k !} \tau^{k-1} \mathrm{e}^{-k \tau},
$$

where

$$
\tau=N t \text {. }
$$

The above expression of $\left\langle n_{k}\right\rangle_{N}$ is quite equal to the solution to the ordinary coagulation equation $N_{k}(\tau)$ (see Eq. (4)). Consequently, we can say that, in the case of $A_{i j}=i \times j,\left\langle n_{k}\right\rangle$ agrees with $N_{k}(\tau)$ when Eqs. (99) and (100) are satisfied. From Eqs. (107) and (108) we see that the value of $\left\langle n_{k}\right\rangle_{N}$ decays rapidly in the course of time and, hence, becomes extremely small at $t \gg 1 / N$. When the constraint (100) is violated, we are not so much interested in $\left\langle n_{k}\right\rangle_{N}$ because of its smallness. Therefore, the constraint (100) is not so essential from the point of practical use.

\section{Concluding Remarks}

The statistical coagulation equation usually used to describe the planetary growth breaks down when the number of particles becomes small in process of the accumulation. In order to overcome this defect, we regarded the planetary growth as the stochastic process and derived the stochastic coagulation equation. Although the stochastic coagulation equation exactly describes the accumulation process, it is hopelessly difficult to solve it for general cases. This difficulty make it important to clarify what conditions are necessary for the stochastic coagulation equation to reduce to the ordinary, statistical coagulation equation. The investigation of the stochastic coagulation equation gave us the following results:

1. The stochastic coagulation equation can reduce to the coagulation equation if $\left\langle n_{i}\left(n_{j}-\delta_{i j}\right)\right\rangle$ is approximated well by $\left\langle n_{i}\right\rangle\left\langle n_{j}\right\rangle$ for all $i$ and $j$. This condition seem to be reasonable but not sufficient to the practical use because we do not know explicitly whether or not the condition is satisfied.

2. For the cases of $A_{i j}=1, i+j$ and $i \times j$, we found the analytic solutions to the stochastic coagulation equation and obtained, for the latter two cases, explicitly the condition under which the coagulation equation is valid: solutions to the stochastic coagulation equation $\left\langle n_{k}\right\rangle_{N}$ coincide well with those to the coagulation equation $N_{k}$, if the mass $k$ satisfies an inequality $k^{2} \ll N$. 
It should be noted that the condition $k^{2} \ll N$ is just the sufficient condition: for the larger $k$, the coagulation equation may be valid in some cases. In order to obtain the necessary and sufficient condition, further comparison between the solutions to the stochastic coagulation equation and the ordinary coagulation equation has to be accomplished. This will be presented in the subsequent paper.

The authors are indebted to Professor T. Shiga for the valuable suggestions. They wish to express their gratitude to K. Ohtsuki and H. Emori for their frequent and enlightening discussions and to T. Yuda for her help in preparing the manuscript. Numerical computations where performed by the ETA ${ }^{10}$ at the Computer Center of Tokyo Institute of Technology. This work was supported partly by a Grant-in-Aid for General Scientific Research (No. 02452062). This is contributions of the Department of Earth and Planetary Sciences, Tokyo Institute of Technology, No. 8.

\section{Appendix A: Property of $g_{\bar{N} l}$}

In this appendix, we will prove that $g_{\bar{N} l}$ given by Eq. (53) is independent of time $t$. Since we assumed that there are $N$ particles having the unit mass initially and that the collision never leads to the fragmentation, there is no possible state that contains particles more than the initial state. That is, the total particle number is not greater than $N$.

First, we consider a state in which there exist $N$ particles. There is no such state other than the initial state owing to the above assumptions. Hence, the probability that the system is in the initial state $\mathbf{n}_{i}$ is equal to the probability that the total particle number is $N$ :

$$
f\left(\mathbf{n}_{i} ; t\right)=F(N ; t) .
$$

By the use of Eq. (53), we obtain

$$
g_{N, 1}=1,
$$

where we labeled the initial state by $(N, 1)$. Thus, when the total particle number $\bar{N}$ is $\mathrm{N}, g_{\bar{N} l}$ is independent of $t$.

Secondly, for a certain integer $\bar{N}_{0}<N$ we make an assumption that $g_{\bar{N}_{0}+1, l}$ is independent of time $t$ (which we call assumption (1) in this appendix). Under the assumption (1) we will prove that $g_{\bar{N}_{0} l}$ is independent of $t$. Using Eq. (53) and noting that the operator $a^{(3)}=a_{k} a_{i}^{\dagger} a_{j}^{\dagger}$ changes a state with $\bar{N}$ particles into a state with $\bar{N}+1$ particles, we can transform the right-hand side of Eq. (39) as

$$
\begin{aligned}
& \frac{1}{2} \sum_{k=1}^{N} \sum_{i, j=1}^{N} A_{i j} \delta_{i+j, k} a_{k} a_{i}^{\dagger} a_{j}^{\dagger} n_{i}\left(n_{j}-\delta_{i j}\right) f(\mathbf{n} ; t) \\
& =\frac{1}{2} \sum_{k=1}^{N} \sum_{i, j=1}^{N} A_{i j} \delta_{i+j, k} a_{k} a_{i}^{\dagger} a_{j}^{\dagger} n_{i}\left(n_{j}-\delta_{i j}\right) F(\bar{N} ; t) g_{\bar{N} l} \\
& =\frac{1}{2} F(\bar{N}+1 ; t) \sum_{k=1}^{N} \sum_{i, j=1}^{N} A_{i j} \delta_{i+j, k} a_{k} a_{i}^{\dagger} a_{j}^{\dagger} n_{i}\left(n_{j}-\delta_{i j}\right) g_{\bar{N} l} \\
& =\zeta(\mathbf{n}) F(\bar{N}+1 ; t)
\end{aligned}
$$

where

$$
\zeta(\mathbf{n})=\frac{1}{2} \sum_{k=1}^{N} \sum_{i, j=1}^{N} A_{i j} \delta_{i+j, k} a_{k} a_{i}^{\dagger} a_{j}^{\dagger} n_{i}\left(n_{j}-\delta_{i j}\right) g_{\bar{N} l} .
$$


Hence, for a state $\mathbf{n}_{0}$ containing $\bar{N}_{0}$ particles Eq. (39) becomes

$$
\left\{\frac{\partial}{\partial t}+\alpha\left(\bar{N}_{0}\right)\right\} f\left(\mathbf{n}_{0} ; t\right)=\zeta\left(\mathbf{n}_{0}\right) F\left(\bar{N}_{0}+1 ; t\right) .
$$

Since the operator $a^{(3)}=a_{k} a_{i}^{\dagger} a_{j}^{\dagger}$ turns $g_{\bar{N}_{0} l}$ into $g_{\bar{N}_{0}+1, l^{\prime}}$, which is independent of $t$ because of the assumption (1), $\zeta\left(\mathbf{n}_{0}\right)$ defined above is also independent of $t$. Thus, Eq. (A.5) has the same form as that of Eq. (44) except for the time-independent coefficient of $F\left(\bar{N}_{0}+1 ; t\right)$ on the right-hand side. Furthermore, since at time $t=0$ both $f\left(\mathbf{n}_{0} ; t\right)$ and $F\left(\bar{N}_{0} ; t\right)$ are equal to zero, we can express the solution to Eq. (A.5) by means of $F\left(\bar{N}_{0} ; t\right)$ :

$$
f\left(\mathbf{n}_{0} ; t\right)=\frac{\zeta\left(\mathbf{n}_{0}\right)}{\alpha\left(\bar{N}_{0}+1\right)} F\left(\bar{N}_{0} ; t\right),
$$

By the comparison with Eq. (53) we have for $g_{\bar{N}_{0} l}$

$$
g_{\bar{N}_{0} l}=\frac{\zeta\left(\mathbf{n}_{0}\right)}{\alpha\left(\bar{N}_{0}+1\right)}
$$

and find that $g_{\vec{N}_{0} l}$ is independent of time $t$ under the assumption (1).

Consequently, by means of the mathematical induction we can conclude that for arbitrary integers $\bar{N} \leq N$ and $l$ (i.e., for all possible state) $g_{\bar{N} l}$ is independent of time $t$.

\section{REFERENCES}

FUjiwara, A. and Y. TAKAgI, Outcomes of direct collision, Prog. Theor. Phys. Suppl., 96, 228-238, 1988.

GREenBerG, R., J. F. WACKER, W. K. HARTMANN, and C. R. CHAPMAN, Planetesimals to Planets: Numerical simulation of collisional evolution, Icarus, 35, 1-26, 1978.

HAYASHI, C., K. NAKAZAWA, and Y. NAKAGAWA, Formation of the solar system, in Protostars and planets II, edited by D. C. Black and M. S. Matthews, 1100pp., The Univ. of Arizona Press, Tucson, 1985.

OHTSUKI, K., Y. NAKAGAWA, and K. NAKAZAWA, Growth of the Earth in nebula gas, Icarus, 75, 552-565, 1988.

SMOLUCHOwSKI, M. V., Drei Vortrage uber Diffusion, Brownsche Molekularbewegung und Koagulation von Kolloidteilchen, Phys. Zeits., 17, 557-571, 585-599, 1916.

TRUBNIKOV, B. A., Solution of the coagulation equations in the case of a bilinear coefficient of adhesion of particles, Sov. Phys. Dokl., 16, 124-126, 1971.

Wetherill, G. W., Formation of the terrestrial planets, Ann. Rev. Aston. Astrophys., 18, 77-113, 1980.

WETHERILL, G. W., Comparison of analytical and physical modeling of planetesimal accumulation, Icarus, 88 , 336-354, 1990.

WETHERILL, G. W. and G. R. STEWART, Accumulation of a swarm of small planetesimals, Icarus, 77, 330-357, 1989. 